\title{
Automorphism group of the derangement graph*
}

\author{
Yun-Ping Deng and Xiao-Dong Zhang ${ }^{\dagger}$ \\ Department of Mathematics \\ Shanghai Jiao Tong University \\ Shanghai 200240, P. R. China \\ dyp612@hotmail.com, xiaodong@sjtu.edu.cn
}

Submitted: Nov 8, 2010; Accepted: Sep 27, 2011; Published: Oct 3, 2011

Mathematics Subject Classifications: 05C25, 05C69

\begin{abstract}
In this paper, we prove that the full automorphism group of the derangement graph $\Gamma_{n}(n \geq 3)$ is equal to $\left(R\left(S_{n}\right) \rtimes \operatorname{Inn}\left(S_{n}\right)\right) \rtimes Z_{2}$, where $R\left(S_{n}\right)$ and $\operatorname{Inn}\left(S_{n}\right)$ are the right regular representation and the inner automorphism group of $S_{n}$ respectively, and $Z_{2}=\langle\varphi\rangle$ with the mapping $\varphi: \sigma^{\varphi}=\sigma^{-1}, \forall \sigma \in S_{n}$. Moreover, all orbits on the edge set of $\Gamma_{n}(n \geq 3)$ are determined.
\end{abstract}

Keywords: derangement graph, automorphism group, Cayley graph, symmetric group

\section{Introduction}

For a finite, simple and undirected graph $\Gamma$, we use $V(\Gamma), E(\Gamma)$ and $\operatorname{Aut}(\Gamma)$ to denote its vertex set, edge set and full automorphism group, respectively. Let $G$ be a finite group and $S$ a subset of $G$ not containing the identity element 1 . The Cayley graph $\Gamma:=\operatorname{Cay}(G, S)$ on $G$ with respect to $S$ is defined by

$$
V(\Gamma)=G, E(\Gamma)=\{(g, s g) \mid g \in G, s \in S\} .
$$

If $S=S^{-1}$, then $\operatorname{Cay}(G, S)$ can be viewed as an undirected graph by identifying an undirected edge $\{g, h\}$ with two directed edges $(g, h)$ and $(h, g)$. It is easy to see from the definition that there are two obvious facts: (1) $\Gamma$ is regular of vertex degree $|S| ;(2) \Gamma$ is connected if and only if $G=\langle S\rangle$.

${ }^{*}$ This work is supported by National Natural Science Foundation of China (No:10971137), the National Basic Research Program (973) of China (No.2006CB805900), and a grant of Science and Technology Commission of Shanghai Municipality (STCSM, No:09XD1402500) .

${ }^{\dagger}$ Correspondent author : Xiao-Dong Zhang 
A bijection of a finite set $\Omega$ to itself is a permutation of $\Omega$. Let $S_{n}$ be the symmetric group of permutations of $X=\{1,2, \cdots, n\}$, and let $\mathcal{D}_{n}:=\left\{\sigma \in S_{n} \mid x^{\sigma} \neq x, \forall x \in X\right\}$ denote the derangements on $X$, namely the set of fixed point free permutations of $S_{n}$. The graph $\Gamma_{n}:=\operatorname{Cay}\left(S_{n}, \mathcal{D}_{n}\right)$ is called the derangement graph on $X$. Moreover, denote $\left|\mathcal{D}_{n}\right|$ by $D_{n}$ for the convenience of writing.

There are many nice structures and properties on the derangement graph $\Gamma_{n}$ which are discovered by researchers. For example, Renteln [17] proved that it is connected for $n \geq 4$, the clique number $\omega\left(\Gamma_{n}\right)=n$, and the chromatic number $\chi\left(\Gamma_{n}\right)=n$. Imrich [13] and Hamidoune [11] independently proved that the vertex connectivity $\kappa\left(\Gamma_{n}\right)=D_{n}$. Eggleton and Wallis [4], and Rasmussen and Savage [16] observed that $\Gamma_{n}$ is Hamiltonian. Deza and Frankl [3] proved that the maximum independent number $\alpha\left(\Gamma_{n}\right)=(n-1)$ !. Moreover, the structure of maximum independent set of $\Gamma_{n}$, namely a coset of the stabilizer of a point, has been determined by several authors $([1,10,15,19])$. Ku and Wong [14] conjectured that $\frac{-D_{n}}{n-1}$ is the smallest eigenvalue of $\Gamma_{n}$, which has been confirmed by Renteln [17]. Deng and Zhang [2] proved that $\frac{n-3}{n-1} D_{n-2}$ is the second largest eigenvalue of $\Gamma_{n}$.

On the other hand, it is interesting and difficult to determine the full automorphism group of a graph. However, there are some known results on the automorphism groups of Cayley graphs with small degree. For example, Godsil [9] gave the automorphism groups of some cubic Cayley graphs. Feng and $\mathrm{Xu}$ [7] determined the automorphism groups of tetravalent Cayley graphs on regular p-groups. Recently, Zhang et al. [22] determined the automorphism groups of cubic Cayley graphs of order $2 p q$. For other results on the automorphism groups of Cayley graphs, we refer the readers to [5, 6, 11, 12, 18, 20, 21]. Motivated by the known results and nice structures of the derangement graph, in this paper, we characterize the full automorphism group of the derangement graph. The main result can be stated as follows:

Theorem 1.1. For $n \geq 3$,

$$
\operatorname{Aut}\left(\Gamma_{n}\right)=\left(R\left(S_{n}\right) \rtimes \operatorname{Inn}\left(S_{n}\right)\right) \rtimes Z_{2},
$$

where $R\left(S_{n}\right)$ and $\operatorname{Inn}\left(S_{n}\right)$ are the right regular representation and the inner automorphism group of $S_{n}$ respectively, and $Z_{2}=\langle\varphi\rangle$ with the mapping $\varphi: \sigma^{\varphi}=\sigma^{-1}, \forall \sigma \in S_{n}$.

The rest of this paper is organized as follows. In Section 2, we gather some definitions and known results needed later. In Section 3, we present the proof of Theorem 1.1, i.e., characterize the full automorphism group of the derangement graph $\Gamma_{n}(n \geq 3)$. In Section 4 , we determine all the edge-orbits of $\Gamma_{n}$, which implies that $\Gamma_{n}$ is not edge-transitive.

\section{Preliminaries}

Let $G$ be a finite group and $\Omega$ a finite set. Suppose that, for each $\alpha \in \Omega$ and $g \in G$, there corresponds a member of $\Omega$, denoted by $\alpha^{g}$. We say that this correspondence defines an action of $G$ on $\Omega$, or $G$ acts on $\Omega$, if the following conditions hold: (i) $\forall \alpha \in \Omega, \alpha^{1}=\alpha$, where 1 is the identity element of $G$; (ii) $\forall \alpha \in \Omega, \forall g, h \in \mathrm{G},\left(\alpha^{g}\right)^{h}=\alpha^{g h}$. Furthermore, 
if $\left\{g \in G: \alpha^{g}=\alpha, \forall \alpha \in \Omega\right\}=1$, we say the action of $G$ on $\Omega$ is faithful, or $G$ acts faithfully on $\Omega$.

The action of $G$ on $\Omega$ induces naturally an equivalence relation $\sim_{G}$ which is defined as follows: $\alpha \sim_{G} \beta$ if and only if $\alpha^{g}=\beta$ for some $g \in G$. The equivalence classes of $\sim_{G}$ are said to be $G$-orbits on $\Omega$. If there is only one $\mathrm{G}$-orbit on $\Omega$, then $\mathrm{G}$ is said to be transitive on $\Omega$, or $\mathrm{G}$ acts transitively on $\Omega$. In particular, a graph $\Gamma$ is said to be vertex-transitive or edge-transitive if $\operatorname{Aut}(\Gamma)$ acts transitively on $V(\Gamma)$ or $E(\Gamma)$ respectively.

For a group $G$, let $\operatorname{Aut}(G), \operatorname{Inn}(G)$ and $R(G)$ be the automorphism group, the inner automorphism group and the right regular representation of $G$, respectively. We need the following known results.

Proposition 2.1. [18, III, Theorem 2.18-2.20] If $n \geq 2$ and $n \neq 6$, then $\operatorname{Aut}\left(S_{n}\right)=$ $\operatorname{Inn}\left(S_{n}\right)$. If $n=6$, then $\left|\operatorname{Aut}\left(S_{6}\right): \operatorname{Inn}\left(S_{6}\right)\right|=2$, and for each $\alpha \in \operatorname{Aut}\left(S_{6}\right) \backslash \operatorname{Inn}\left(S_{6}\right)$, $\alpha$ maps a transposition to a product of three disjoint transpositions.

Proposition 2.2. [8] Let $N_{\text {Aut }(\operatorname{Cay}(G, S)}(R(G))$ be the normalizer of $R(G)$ in $\operatorname{Aut}(\operatorname{Cay}(G, S))$. Then

$$
N_{\text {Aut }(\operatorname{Cay}(G, S)}(R(G))=R(G) \rtimes \operatorname{Aut}(G, S) \leq \operatorname{Aut}(\operatorname{Cay}(G, S)),
$$

where $\operatorname{Aut}(G, S)=\left\{\phi \in \operatorname{Aut}(G) \mid S^{\phi}=S\right\}$.

Proposition 2.3. [1] All the maximum-size independent sets of the derangement graph $\Gamma_{n}(n \geq 2)$ are $B_{i, j}=\left\{\sigma \in S_{n} \mid i^{\sigma}=j\right\}, i, j=1,2, \cdots, n$.

\section{Proof of Theorem 1.1}

In this section, we completely determine the full automorphism group of the derangement graph.

Lemma 3.1. Let $B=\left\{B_{i, j} \mid i, j=1,2, \cdots, n\right\}$ with $B_{i, j}=\left\{\sigma \in S_{n} \mid i^{\sigma}=j\right\}$. Then $\operatorname{Aut}\left(\Gamma_{n}\right)$ induces an action on $B$ and this action is faithful. In particular, any $\phi \in \operatorname{Aut}\left(\Gamma_{n}\right)$ is a permutation on $B$.

Proof. Obviously, any $\phi \in \operatorname{Aut}\left(\Gamma_{n}\right)$ maps a maximum-size independent set of $\Gamma_{n}$ to a maximum-size independent set of $\Gamma_{n}$. So by Proposition 2.3, for any $B_{i, j} \in B$ and $\phi \in$ $\operatorname{Aut}\left(\Gamma_{n}\right)$, we have $B_{i, j}^{\phi} \in B$.

Next if $\phi \in \operatorname{Aut}\left(\Gamma_{n}\right)$ satisfies $B_{i, j}^{\phi}=B_{i, j}$ for each $B_{i, j} \in B$, then $\phi$ is the identity map. In fact,

$$
\forall \sigma \in S_{n},\{\sigma\}=B_{1,1^{\sigma}} \cap B_{2,2^{\sigma}} \cap \cdots \cap B_{n, n^{\sigma}}
$$

So

$$
\begin{aligned}
\left\{\sigma^{\phi}\right\} & =\left(B_{1,1^{\sigma}} \cap B_{2,2^{\sigma}} \cap \cdots \cap B_{n, n^{\sigma}}\right)^{\phi} \\
& \subseteq B_{1,1^{\sigma}}^{\phi} \cap B_{2,2^{\sigma}}^{\phi} \cap \cdots \cap B_{n, n^{\sigma}}^{\phi} \\
& =B_{1,1^{\sigma}} \cap B_{2,2^{\sigma}} \cap \cdots \cap B_{n, n^{\sigma}} \\
& =\{\sigma\} .
\end{aligned}
$$


Thus $\phi$ is the identity map, that is, $\operatorname{Aut}\left(\Gamma_{n}\right)$ acts faithfully on $B$. This implies that each $\phi \in \operatorname{Aut}\left(\Gamma_{n}\right)$ is a permutation on $B$.

Lemma 3.2. Let $R_{k}=\left\{B_{k, 1}, B_{k, 2}, \cdots, B_{k, n}\right\}$ and $C_{l}=\left\{B_{1, l}, B_{2, l}, \cdots, B_{n, l}\right\}$. For any $x_{1}, x_{2}, \cdots, x_{n} \in B$, if $x_{1} \cup x_{2} \cup \cdots \cup x_{n}=S_{n}$, then there exists some $k$ or $l \in\{1,2, \cdots, n\}$ such that $\left\{x_{1}, x_{2}, \cdots, x_{n}\right\}=R_{k}$ or $C_{l}$.

Proof. First we claim that $B_{i, j} \cap B_{i^{\prime}, j^{\prime}}=\emptyset$ if and only if exactly one of $i=i^{\prime}$ and $j=j^{\prime}$ holds. In fact, If exactly one of $i=i^{\prime}$ and $j=j^{\prime}$ holds, then $B_{i, j} \cap B_{i^{\prime}, j^{\prime}}=\emptyset$. If $i \neq i^{\prime}$ and $j \neq j^{\prime}$, then $B_{i, j} \cap B_{i^{\prime}, j^{\prime}}=\left\{\sigma \in S_{n} \mid i^{\sigma}=j\right.$ and $\left.i^{\prime \sigma}=j^{\prime}\right\} \neq \emptyset$. If $i=i^{\prime}$ and $j=j^{\prime}$, then $B_{i, j}=B_{i^{\prime}, j^{\prime}}$, so $B_{i, j} \cap B_{i^{\prime}, j^{\prime}} \neq \emptyset$.

Note that $\forall i,\left|x_{i}\right|=(n-1)$ ! and $\left|S_{n}\right|=n$ !. Hence

$$
x_{1} \cup x_{2} \cup \cdots \cup x_{n}=S_{n} \Rightarrow x_{i} \cap x_{j}=\emptyset, \forall i, j, i \neq j .
$$

Applying the above claim, we obtain $\left\{x_{1}, x_{2}, \cdots, x_{n}\right\}=R_{k}$ or $C_{l}$.

Lemma 3.3. Let $\Omega=\left\{R_{1}, R_{2}, \cdots, R_{n}, C_{1}, C_{2}, \cdots, C_{n}\right\}$. Then Aut $\left(\Gamma_{n}\right)$ induces an action on $\Omega$ and this action is faithful. In particular, any $\phi \in \operatorname{Aut}\left(\Gamma_{n}\right)$ is a permutation on $\Omega$.

Proof. First for any $R_{k} \in \Omega$ and $\phi \in \operatorname{Aut}\left(\Gamma_{n}\right)$,

$$
\begin{gathered}
R_{k}=\left\{B_{k, 1}, B_{k, 2}, \cdots, B_{k, n}\right\} \Rightarrow R_{k}^{\phi}=\left\{B_{k, 1}^{\phi}, B_{k, 2}^{\phi}, \cdots, B_{k, n}^{\phi}\right\}, \\
B_{k, 1}^{\phi} \cup B_{k, 2}^{\phi} \cup \cdots \cup B_{k, n}^{\phi}=\left(B_{k, 1} \cup B_{k, 2} \cup \cdots \cup B_{k, n}\right)^{\phi}=S_{n}^{\phi}=S_{n} .
\end{gathered}
$$

By Lemma 3.2, we have $R_{k}^{\phi} \in \Omega$.

Similarly, for any $C_{l} \in \Omega$ and $\phi \in \operatorname{Aut}\left(\Gamma_{n}\right), C_{l}^{\phi} \in \Omega$.

Next suppose that $\phi \in \operatorname{Aut}\left(\Gamma_{n}\right)$ satisfies $R_{k}^{\phi}=R_{k}$ and $C_{l}^{\phi}=C_{l}$ for any $k, l \in$ $\{1,2, \cdots, n\}$. To prove the Lemma, it suffices to show that $\phi$ is the identity map.

Note that

$$
\forall B_{i, j} \in B,\left\{B_{i, j}\right\}=R_{i} \cap C_{j}
$$

So

$$
\left\{B_{i, j}^{\phi}\right\}=\left(R_{i} \cap C_{j}\right)^{\phi} \subseteq R_{i}^{\phi} \cap C_{j}^{\phi}=R_{i} \cap C_{j}=\left\{B_{i, j}\right\} .
$$

By Lemma 3.1, $\phi$ is the identity map, that is, $\operatorname{Aut}\left(\Gamma_{n}\right)$ acts faithfully on $\Omega$. This implies that each $\phi \in \operatorname{Aut}\left(\Gamma_{n}\right)$ is a permutation on $\Omega$.

Lemma 3.4. Let $R=\left\{R_{1}, R_{2}, \cdots, R_{n}\right\}$ and $C=\left\{C_{1}, C_{2}, \cdots, C_{n}\right\}$. For any $\phi \in \operatorname{Aut}\left(\Gamma_{n}\right)$, the following (i)-(ii) hold:

(i) There exists some $i$ such that $R_{i}^{\phi} \in R$ if and only if $R_{i}^{\phi} \in R$ for any $i$;

(ii) There exists some $j$ such that $C_{j}^{\phi} \in C$ if and only if $C_{j}^{\phi} \in C$ for any $j$.

Proof. (i) Suppose that there exist $i, j(\neq i) \in\{1,2, \cdots, n\}$ such that $R_{i}^{\phi} \in R$ and $R_{j}^{\phi} \in C$. Note that

$$
R_{i} \cap R_{j}=\emptyset \text { if } i \neq j \text { and }\left|R_{k} \cap C_{l}\right|=1 \text { for any } k, l \text {. }
$$


So

$$
\left|R_{i} \cup R_{j}\right|=2 n \Rightarrow\left|R_{i}^{\phi} \cup R_{j}^{\phi}\right|=\left|\left(R_{i} \cup R_{j}\right)^{\phi}\right|=2 n .
$$

On the other hand,

$$
R_{i}^{\phi} \in R, R_{j}^{\phi} \in C \Rightarrow\left|R_{i}^{\phi} \cap R_{j}^{\phi}\right|=1 \Rightarrow\left|R_{i}^{\phi} \cup R_{j}^{\phi}\right|=2 n-1,
$$

which is a contradiction. Thus the assertion holds.

(ii) is similar to the proof of (i).

\section{Lemma 3.5.}

$$
\left|\operatorname{Aut}\left(\Gamma_{n}\right)\right| \leq 2(n !)^{2}
$$

Proof. By Lemma 3.3, any $\phi \in \operatorname{Aut}\left(\Gamma_{n}\right)$ is a permutation of $\Omega$. Using Lemma 3.4, we obtain the following disjoint alternatives:

(i) $R^{\phi}=R$ and $C^{\phi}=C$;

(ii) $R^{\phi}=C$ and $C^{\phi}=R$.

So we have

$$
\operatorname{Aut}\left(\Gamma_{n}\right)=\left\{\phi \in \operatorname{Aut}\left(\Gamma_{n}\right) \mid R^{\phi}=R, C^{\phi}=C\right\} \cup\left\{\phi \in \operatorname{Aut}\left(\Gamma_{n}\right) \mid R^{\phi}=C, C^{\phi}=R\right\} .
$$

Hence

$$
\begin{aligned}
\left|\operatorname{Aut}\left(\Gamma_{n}\right)\right| & \leq\left|\left\{\phi \in \operatorname{Aut}\left(\Gamma_{n}\right) \mid R^{\phi}=R, C^{\phi}=C\right\}\right|+\left|\left\{\phi \in \operatorname{Aut}\left(\Gamma_{n}\right) \mid R^{\phi}=C, C^{\phi}=R\right\}\right| \\
& \leq(n !)^{2}+(n !)^{2} \\
& =2(n !)^{2} .
\end{aligned}
$$

Thus the assertion holds.

Now we are ready to prove the main result.

Proof of Theorem 1.1. First we show that the mapping $\varphi: S_{n} \rightarrow S_{n}$ defined as $\sigma^{\varphi}=\sigma^{-1}$ is an automorphism of $\Gamma_{n}$. In fact, obviously, $\varphi$ is a bijection between $S_{n}$ and $S_{n}$. Moreover,

$$
\begin{aligned}
(\sigma, \tau) \in E\left(\Gamma_{n}\right) & \Leftrightarrow \forall i \in\{1,2, \cdots, n\}, i^{\sigma} \neq i^{\tau} \\
& \Leftrightarrow \forall i \in\{1,2, \cdots, n\},\left(i^{\sigma^{-1}}\right)^{\sigma} \neq\left(i^{\sigma^{-1}}\right)^{\tau} \\
& \Leftrightarrow \forall i \in\{1,2, \cdots, n\}, i \neq i^{\sigma^{-1} \tau} \\
& \Leftrightarrow \forall i \in\{1,2, \cdots, n\}, i^{\tau^{-1}} \neq\left(i^{\sigma^{-1} \tau}\right)^{\tau^{-1}} \\
& \Leftrightarrow \forall i \in\{1,2, \cdots, n\}, i^{\tau^{-1}} \neq i^{\sigma^{-1}} \\
& \Leftrightarrow\left(\sigma^{\varphi}, \tau^{\varphi}\right)=\left(\sigma^{-1}, \tau^{-1}\right) \in E\left(\Gamma_{n}\right) .
\end{aligned}
$$

This implies that $\varphi$ is an automorphism of $\Gamma_{n}$.

Next we claim that $R\left(S_{n}\right) \rtimes \operatorname{Inn}\left(S_{n}\right) \leq \operatorname{Aut}\left(\Gamma_{n}\right)$. In fact, by Proposition 2.1, we have

$$
\operatorname{Aut}\left(S_{n}, \mathcal{D}_{n}\right)=\left\{\phi \in \operatorname{Aut}\left(S_{n}\right) \mid \mathcal{D}_{n}^{\phi}=\mathcal{D}_{n}\right\}=\operatorname{Inn}\left(S_{n}\right),
$$


where $\mathcal{D}_{n}=\left\{\sigma \in S_{n} \mid x^{\sigma} \neq x, \forall x \in\{1,2, \cdots, n\}\right\}$. Then by Proposition 2.2, $R\left(S_{n}\right) \rtimes$ $\operatorname{Inn}\left(S_{n}\right) \leq \operatorname{Aut}\left(\Gamma_{n}\right)$.

Note that $S_{n}(n \geq 3)$ is a centerless group. $\operatorname{So} \operatorname{Inn}\left(S_{n}\right) \cong S_{n} / Z\left(S_{n}\right)=S_{n}$, where $Z\left(S_{n}\right)$ is the center of $S_{n}$. Thus $(n !)^{2}=\left|R\left(S_{n}\right) \rtimes \operatorname{Inn}\left(S_{n}\right)\right| \leq\left|\operatorname{Aut}\left(\Gamma_{n}\right)\right| \leq 2(n !)^{2}$ (by Lemma 3.5), that is, the index of $R\left(S_{n}\right) \rtimes \operatorname{Inn}\left(S_{n}\right)$ in $\operatorname{Aut}\left(\Gamma_{n}\right)$ is at most 2, which implies that $R\left(S_{n}\right) \rtimes \operatorname{Inn}\left(S_{n}\right)$ is a normal subgroup of $\operatorname{Aut}\left(\Gamma_{n}\right)$. In addition, it is easy to see that $\varphi \notin R\left(S_{n}\right)$ and $\varphi \notin \operatorname{Inn}\left(S_{n}\right)$ for $n \geq 3$. Hence $\left(R\left(S_{n}\right) \rtimes \operatorname{Inn}\left(S_{n}\right)\right) \rtimes Z_{2} \leq \operatorname{Aut}\left(\Gamma_{n}\right)$ (where $Z_{2}=\langle\varphi\rangle$ is a cyclic group of order 2) and $2(n !)^{2}=\left|\left(R\left(S_{n}\right) \rtimes \operatorname{Inn}\left(S_{n}\right)\right) \rtimes Z_{2}\right| \leq\left|\operatorname{Aut}\left(\Gamma_{n}\right)\right| \leq$ $2(n !)^{2}$, which shows that $\operatorname{Aut}\left(\Gamma_{n}\right)=\left(R\left(S_{n}\right) \rtimes \operatorname{Inn}\left(S_{n}\right)\right) \rtimes Z_{2}$. The assertion holds.

\section{Edge-orbits of the derangement graph}

It is well known that any permutation can be decomposed as a product of disjoint permutation cycles. For any $\sigma \in S_{n}$, write

$$
\sigma=\left(a_{11} a_{12} \cdots a_{1 n_{1}}\right)\left(a_{21} \cdots a_{2 n_{2}}\right) \cdots\left(a_{s 1} \cdots a_{s n_{s}}\right)
$$

a product of disjoint cycles (including 1-cycles), with $n_{1} \geq n_{2} \geq \cdots \geq n_{s}$ and $n_{1}+n_{2}+$ $\cdots+n_{s}=n$, and we call $\left(n_{1}, n_{2}, \cdots, n_{s}\right)$ the cycle-shape of $\sigma$.

For any $\sigma \in S_{n}$ and $\phi \in \operatorname{Inn}\left(S_{n}\right) \times Z_{2}$ (where $Z_{2}=\langle\varphi\rangle$ is same as Theorem 1.1), obviously $\sigma$ and $\sigma^{\phi}$ have the same cycle-shape. Note that $\varphi$ commutes with each element in $\operatorname{Inn}\left(S_{n}\right)$. Thus, $\operatorname{Inn}\left(S_{n}\right) \rtimes Z_{2}=\operatorname{Inn}\left(S_{n}\right) \times Z_{2}$.

Lemma 4.1. Let $\operatorname{Aut}\left(\Gamma_{n}\right)(n \geq 3)$ act naturally on $E\left(\Gamma_{n}\right)$. For any $(1, \tau),(1, \sigma) \in E\left(\Gamma_{n}\right)$, $(1, \tau)$ and $(1, \sigma)$ belong to the same Aut $\left(\Gamma_{n}\right)$-orbit if and only if $\tau$ and $\sigma$ have the same cycle-shape.

Proof. $(\Rightarrow)$ If $(1, \tau)$ and $(1, \sigma)$ belong to the same Aut $\left(\Gamma_{n}\right)$-orbit, then we have the following disjoint alternatives:

(i) There exists $\phi \in \operatorname{Aut}\left(\Gamma_{n}\right)$ such that $1^{\phi}=1$ and $\tau^{\phi}=\sigma$;

(ii) There exists $\phi \in \operatorname{Aut}\left(\Gamma_{n}\right)$ such that $\tau^{\phi}=1$ and $1^{\phi}=\sigma$.

By Theorem 1.1, we can always assume that $\phi=R(g) \cdot \phi^{\prime}$, where $\phi^{\prime} \in \operatorname{Inn}\left(S_{n}\right) \times Z_{2}$.

If the case (i) happens, then

$$
\begin{gathered}
1^{\phi}=1 \Rightarrow 1^{R(g) \cdot \phi^{\prime}}=1 \Rightarrow g^{\phi^{\prime}}=1 \Rightarrow g=1 . \\
\tau^{\phi}=\sigma \Rightarrow \tau^{R(g) \cdot \phi^{\prime}}=\sigma \Rightarrow(\tau g)^{\phi^{\prime}}=\sigma \Rightarrow \tau^{\phi^{\prime}}=\sigma .
\end{gathered}
$$

So $\tau$ and $\sigma$ have the same cycle-shape.

If the case (ii) happens, then

$$
\begin{gathered}
\tau^{\phi}=1 \Rightarrow \tau^{R(g) \cdot \phi^{\prime}}=1 \Rightarrow(\tau g)^{\phi^{\prime}}=1 \Rightarrow \tau g=1 \Rightarrow g=\tau^{-1} . \\
1^{\phi}=\sigma \Rightarrow 1^{R(g) \cdot \phi^{\prime}}=\sigma \Rightarrow g^{\phi^{\prime}}=\sigma \Rightarrow\left(\tau^{-1}\right)^{\phi^{\prime}}=\sigma .
\end{gathered}
$$

So $\tau^{-1}$ and $\sigma$ have the same cycle-shape, that is, $\tau$ and $\sigma$ have the same cycle-shape.

$(\Leftarrow)$ If $\tau$ and $\sigma$ have the same cycle-shape, then there exists some $\phi \in \operatorname{Inn}\left(S_{n}\right) \leq$ $\operatorname{Aut}\left(\Gamma_{n}\right)$ such that $\tau^{\phi}=\sigma$. Hence $(1, \tau)^{\phi}=\left(1^{\phi}, \tau^{\phi}\right)=(1, \sigma)$, that is, $(1, \tau)$ and $(1, \sigma)$ belong to the same $\operatorname{Aut}\left(\Gamma_{n}\right)$-orbit. 
Using Lemma 4.1, we have the following result:

Corollary 4.2. Let $\operatorname{Aut}\left(\Gamma_{n}\right)(n \geq 3)$ act naturally on $E\left(\Gamma_{n}\right)$. For any $\left(\sigma_{1}, \tau_{1}\right),\left(\sigma_{2}, \tau_{2}\right) \in$ $E\left(\Gamma_{n}\right),\left(\sigma_{1}, \tau_{1}\right)$ and $\left(\sigma_{2}, \tau_{2}\right)$ belong to the same Aut $\left(\Gamma_{n}\right)$-orbit if and only if $\tau_{1} \sigma_{1}^{-1}$ and $\tau_{2} \sigma_{2}^{-1}$ have the same cycle-shape.

Proof. $\left(\sigma_{1}, \tau_{1}\right)$ and $\left(\sigma_{2}, \tau_{2}\right)$ belong to the same Aut $\left(\Gamma_{n}\right)$-orbit $\Leftrightarrow$ there exists some $\phi \in$ $\operatorname{Aut}\left(\Gamma_{n}\right)$ such that $\left(\sigma_{1}, \tau_{1}\right)^{\phi}=\left(\sigma_{2}, \tau_{2}\right) \Leftrightarrow\left(1, \tau_{1} \sigma_{1}^{-1}\right)^{R\left(\sigma_{1}\right) \phi R\left(\sigma_{2}^{-1}\right)}=\left(1, \tau_{2} \sigma_{2}^{-1}\right) \Leftrightarrow\left(1, \tau_{1} \sigma_{1}^{-1}\right)$ and $\left(1, \tau_{2} \sigma_{2}^{-1}\right)$ belong to the same $\operatorname{Aut}\left(\Gamma_{n}\right)$-orbit. By Lemma 4.1, the assertion holds.

By the definition of the derangement graph $\Gamma_{n}$, we have $(\sigma, \tau) \in E\left(\Gamma_{n}\right)$ if and only if $\tau \sigma^{-1} \in \mathcal{D}_{n}$. Therefore, applying Corollary 4.2, the Aut $\left(\Gamma_{n}\right)$-orbits on $E\left(\Gamma_{n}\right)$ are in bijective correspondence with the set of all possible cycle-shapes of permutations in $\mathcal{D}_{n}$. So we obtain the main result in this section as follows:

Theorem 4.3. Let $\operatorname{Aut}\left(\Gamma_{n}\right)(n \geq 3)$ act naturally on $E\left(\Gamma_{n}\right)$. All Aut $\left(\Gamma_{n}\right)$-orbits are $O_{\left(n_{1}, n_{2}, \cdots, n_{s}\right)}=\left\{(\sigma, \tau) \in E\left(\Gamma_{n}\right) \mid \tau \sigma^{-1}\right.$ has cycle-shape $\left.\left(n_{1}, n_{2}, \cdots, n_{s}\right), n_{s} \geq 2\right\}$. In particular, the number of edge-orbits of $\Gamma_{n}(n \geq 3)$ equals to the cardinality of the set $\left\{\left\{n_{1}, n_{2}, \cdots, n_{s}\right\} \mid n=n_{1}+n_{2}+\cdots+n_{s}, n_{i} \geq 2,1 \leq i \leq s\right\}$.

\section{Acknowledgements}

The authors would like to thank the anonymous referees very much for valuable suggestions, corrections and comments which result in a great improvement of the original manuscript.

\section{References}

[1] P. J. Cameron and C. Y. Ku, Intersecting families of permutations, European J. Combin. 24 (2003), 881-890.

[2] Y.-P. Deng and X.-D. Zhang, A note on eigenvalues of the derangement graph, Ars Combin. 101 (2011), 289-299.

[3] M. Deza and P. Frank, On the maximum number of permutations given maximal or minimal distance, J. Combin. Theory Ser. A 22 (1977), 352-360.

[4] R. B. Eggleton and W. D. Wallis, Problem 86: solution I, Math. Mag. 58 (1985), $112-113$.

[5] X. G. Fang, C. E. Praeger and J. Wang, On the automorphism groups of Cayley graphs of finite simple groups, J. London Math. Soc. (2) 66 (2002), 563-578.

[6] Y. Q. Feng, Automorphism groups of Cayley graphs on symmetric groups with generating transposition sets, J. Combin. Theory Ser. B 96 (2006), 67-72.

[7] Y. Q. Feng and M. Y. Xu, Automorphism groups of tetravalent Cayley graphs on regular p-groups, Discrete Math. 305 (2005), 354-360. 
[8] C. D. Godsil, On the full automorphism group of a graph, Combinatorica 1 (1981), $243-256$.

[9] C. D. Godsil, The automorphism groups of some cubic Cayley graphs, European J. Combin. 4 (1983), 25-32.

[10] C. Godsil and K. Meagher, A new proof of the Erdős-Ko-Rado theorem for intersecting families of permutations, European J. Combin. 30(2009), 404-414.

[11] Y. O. Hamidoune, On the connectivity of Cayley digraphs, European J. Combin. 5 (1984), 309-312.

[12] H. L. Huan, H. M. Liu and W. Xie, Automorphism groups of a family of Cayley graphs on alternating groups, Journal of Systems Science and Information 5 (2007), $37-42$.

[13] W. Imrich, On the connectivity of Cayley graphs, J. Combin. Theory Ser. B 26 (1979), 323-326.

[14] C. Y. Ku and T. W. H. Wong, Intersecting families in the alternating group and direct product of symmetric groups, Electron. J. Combin. 14 (2007), \#R25.

[15] B. Larose and C. Malvenuto, Stable sets of maximal size in Kneser-type graphs, European J. Combin. 25 (2004), 657-673.

[16] D. J. Rasmussen and C. D. Savage, Hamilton-Connected Derangement Graphs on $S_{n}$, Discrete Math. 133 (1994), 217-223.

[17] P. Renteln, On the Spectrum of the Derangement Graph, Electron. J. Combin. 14 (2007), \#R82.

[18] M. Suzuki, Group theory I, Springer, New York, 1982.

[19] J. Wang and S. J. Zhang, An Erdős-Ko-Rado-type theorem in Coxeter groups, European J. Combin. 29 (2008), 1112-1115.

[20] M. Y. Xu, Automorphism groups and isomorphisms of Cayley digraphs, Discrete Math. 182 (1998), 309-319.

[21] Z. Zhang and Q. X. Huang, Automorphism group of bubble-sort graphs and modified bubble-sort graphs, Adv. Math. (China) 34 (2005), 441-447.

[22] C. Zhang, J. X. Zhou and Y. Q. Feng, Automorphisms of cubic Cayley graphs of order 2pq, Discrete Math. 309 (2009), 2687-2695. 